\title{
Update on NASA's Laser Communications Relay Demonstration Project
}

\author{
Bernard L. Edwards, ${ }^{1}$ and David J. Israel ${ }^{2}$ \\ NASA Goddard Space Flight Center, Greenbelt, MD 20771, USA
}

\begin{abstract}
This paper provides an update on NASA's Laser Communications Relay Demonstration Project (LCRD), a joint project between NASA's Goddard Space Flight Center (GSFC), the Jet Propulsion Laboratory, California Institute of Technology (JPL), and the Massachusetts Institute of Technology Lincoln Laboratory (MIT/LL). LCRD will provide a minimum of two years of high data rate optical communications services to demonstrate a concept of operations for future mission critical Earth relay satellites. LCRD is expected to launch in June 2019 and demonstrate how optical communications can meet NASA's growing need for higher data rates, or for the same data rate provided by a comparable RF system, how it enables lower power, lower mass communications systems on user spacecraft. In addition, LCRD's architecture will allow it to serve as a testbed in space for the development of additional symbol coding, link and network layer protocols, etc. LCRD's mission and architecture has slowly evolved since the project first started and this paper will provide an update of LCRD and planned capabilities and experiments.
\end{abstract}

\section{Introduction}

$\mathrm{T}$ HE Laser Communications Relay Demonstration (LCRD) project is NASA's Near Earth flagship optical communications project and is scheduled to begin demonstrations in 2019. The LCRD flight payload consists of two optical communications terminals and the associated electronics and will fly on a geosynchronous satellite. Bidirectional user data rates up to 1.244 gigabits per second are supported. The host spacecraft is being augmented with a high data rate radio frequency (RF) system to support LCRD demonstrations and operations. The LCRD ground segment consists of two optical ground stations and a control center. The two optical ground stations will function as both an endpoint for the optical relay trunk lines and as simulated optical relay users.

LCRD will demonstrate:

- High rate bi-directional communications between Earth and Geostationary Earth Orbit (GEO)

- Real-time optical relay from Optical Ground Station 1 on Earth through the GEO flight payload to Optical Ground Station 2 on Earth

- Differential Phase Shift Keying Modulations suitable for Near Earth high data rate communications

- Pulse Position Modulations suitable for deep space communications or other power limited users, such as small Near Earth missions

- Demonstration of various mission scenarios through spacecraft simulations at the Earth ground stations

- Performance testing and demonstrations of coding, link layer, and network layer protocols over the optical and high data rate RF links

The LCRD flight payload was designed to also be able to communicate with user terminals in Low Earth Orbit (LEO). NASA Goddard Space Flight Center and MIT Lincoln Laboratory are currently developing an optical communications terminal for the International Space Station to operate with LCRD. Thus the LCRD flight payload has a requirement to be able to support high rate bi-directional communications between LEO and GEO as well as between Earth and GEO. Over time, LCRD's requirements and capabilities have been increased, enabling LCRD to serve as a technology demonstrator and as a bridge to NASA's future operational network [1]. For example, LCRD's life expectancy has been increased so it can provide residual operational support to NASA missions after the initial two-year technology demonstration. In addition to the key redundancy added to increase its life expectancy, a high-

\footnotetext{
${ }^{1}$ Chief Communications Systems Engineer, Electrical Engineering Division

${ }^{2}$ Space Communications Architect, Exploration and Space Communications Projects Division
} 
bandwidth Ka-band radio frequency (RF) link is being added to the host spacecraft to demonstrate a future concept of operations for a relay satellite with both optical and RF services. The latest LCRD mission architecture is depicted in Figure 1. The purpose of this paper is to bring the reader up to date with the most important aspects of the project.

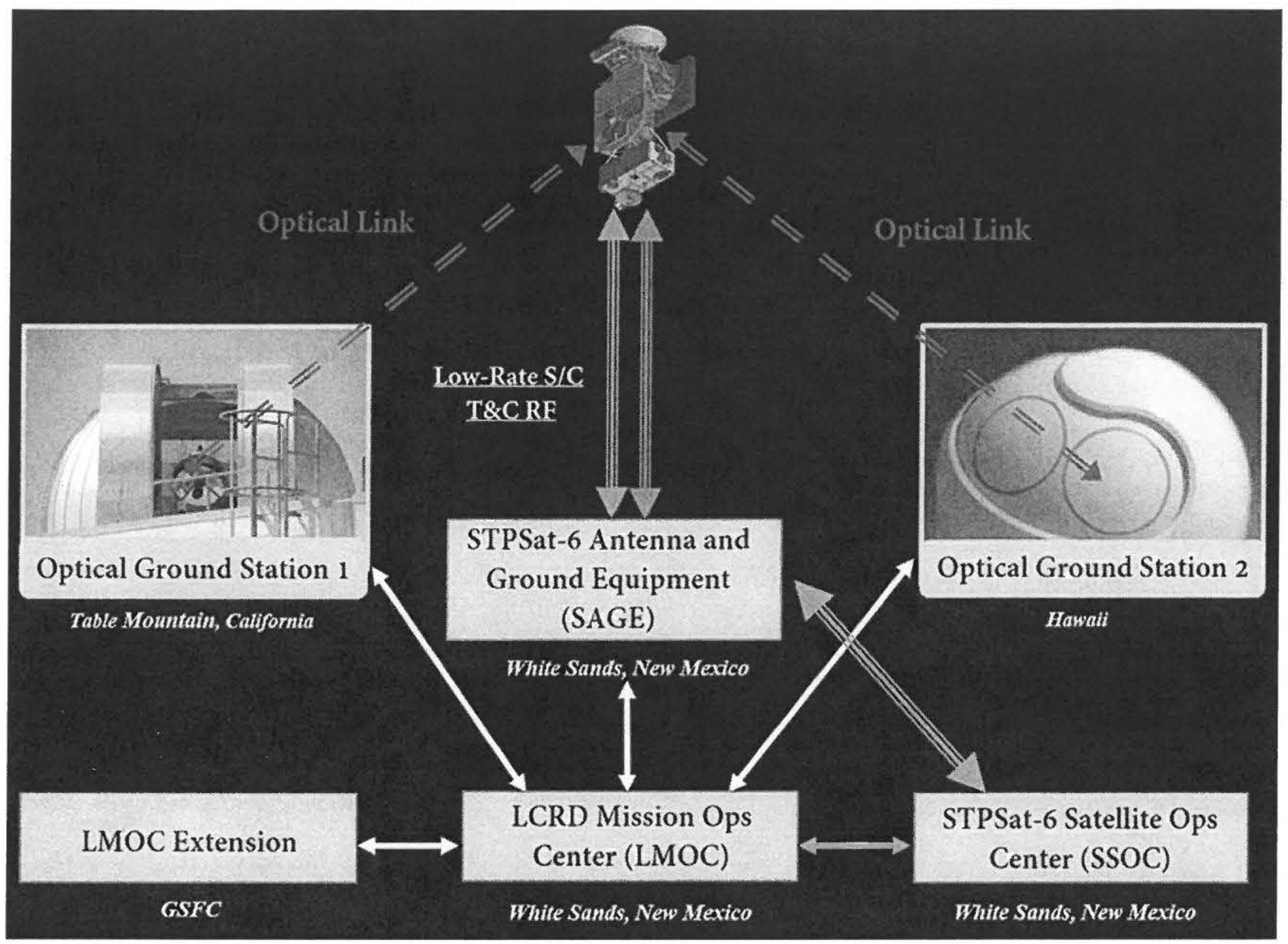

Fig. 1 LCRD Mission Architecture

\section{The Flight Payload}

The LCRD flight payload will be flown on a GEO spacecraft and consists of:

- Two optical communications modules (heads)

- Two optical module controllers

- Two modems capable of supporting both Differential Phase Shift Keying (DPSK) and Pulse Position Modulation (PPM)

- Two space switching units (one is a spare) to interconnect the two optical modules and the high data rate RF system

- A secure host interface module (SHIM) to interface to the host spacecraft

An optical communications terminal on LCRD consists of an optical module, a modem, and an optical module controller.

\section{A. Optical Module}

Each optical module, shown in Figure 2, is a 4-inch reflective telescope that produces a $\sim 15$ microradian downlink beam. It also houses a spatial acquisition detector which is a simple quadrant detector, with a field of view of approximately 2 microradians. It is used both for detection of a scanned uplink signal, and as a tracking sensor for 
initial pull-in of the signal. The telescope is mounted to a two-axis gimbal via a magnetohydrodynamic inertial reference unit (MIRU). Angle-rate sensors in the MIRU detect angular disturbances which are then rejected using voice-coil actuators for inertial stabilization of the telescope. Optical fibers couple the optical module to the modems where transmitted optical waveforms are processed. Control for each optical module and its corresponding modems are provided by a controller. Each optical module is held and protected during launch with a cover and one-time launch latch.

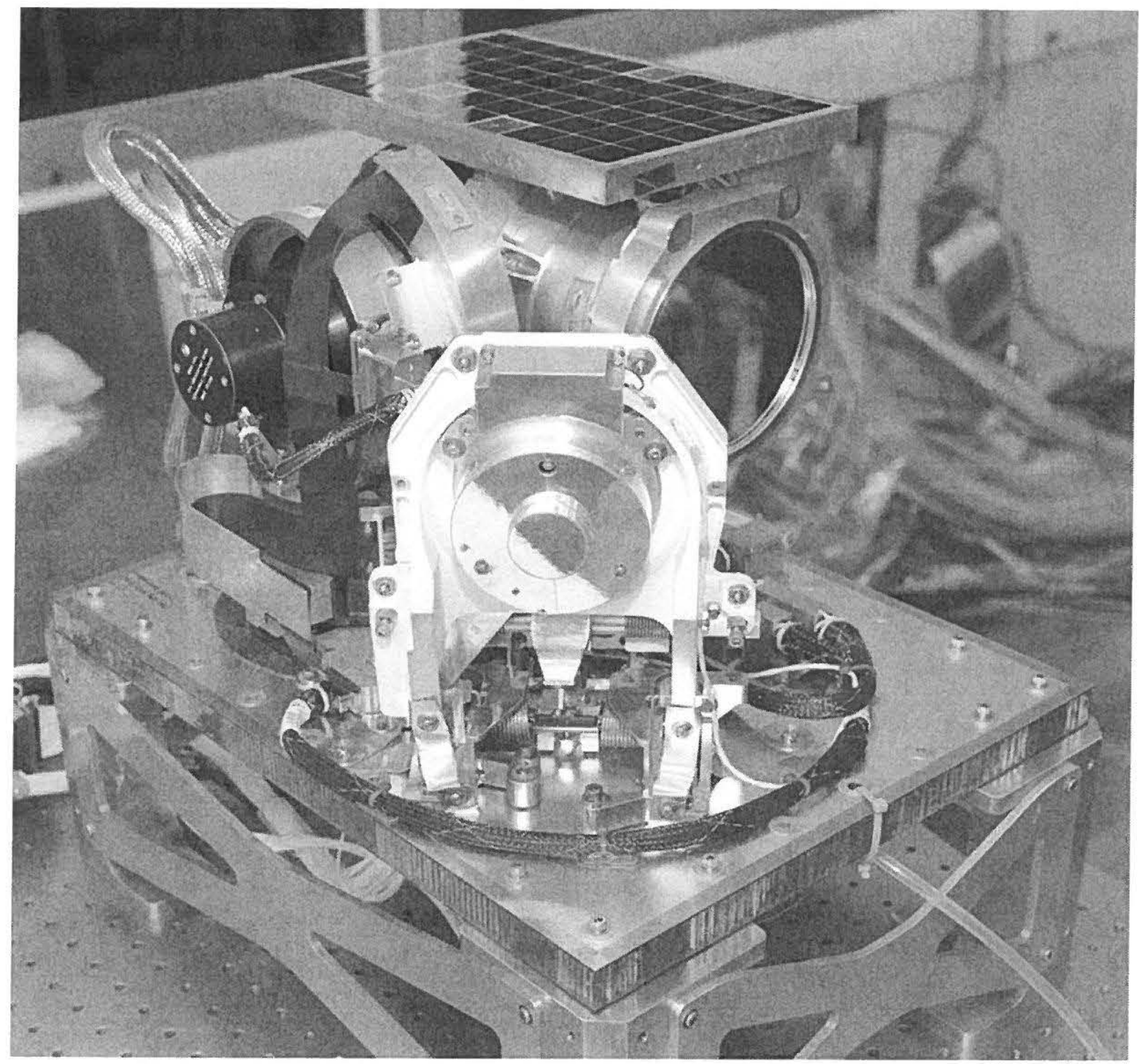

Fig. 2 Optical Module 1

\section{B. Flight Modem}

LCRD's primary modulation format is Differential Phase Shift Keying (DPSK) which has superior noise tolerance, can be used at extremely high data rates, and supports communications when the Sun is in the field of view. LCRD leverages a MIT/LL previously designed DPSK modem [3]. It can both transmit and receive data at an (uncoded) rate from $72 \mathrm{Mbps}$ to $2.88 \mathrm{Gbps}$. The modem employs identical signaling for both the uplink and downlink directions. The DPSK transmitter generates a sequence of pulses at a $2.88 \mathrm{GHz}$ clock rate. A bit is encoded in the phase difference between consecutive pulses. As demodulation is accomplished with a Mach-Zehnder optical interferometer, the clock rate remains fixed. The DPSK transmitter utilizes a MOPA architecture [3]. The EDFA amplifies the optical signal to a 0.5 -W average power level. Data rates below the maximum are accomplished via "burst-mode" operation, where the transmitter sends pulses only a fraction of the time, sending no optical power the remainder of the time. Since the 
EDFA is average power limited, the peak power during the bursts is increased; thus the rate reduction is accomplished in a power efficient manner.

The DPSK receiver has an optical pre-amplifier stage and an optical filter, at which point the light is split between a clock recovery unit and the communications receiver. The receiver uses a delay-line interferometer followed by balanced photo-detectors to compare the phases of consecutive pulses, making a hard decision on each channiel bit. While coding and interleaving will be applied in the ground terminal to mitigate noise and atmospheric fading, the DPSK flight receiver does not decode nor de-interleave. The modems instead support a relay architecture where upand down-link errors are corrected together in a decoder located at the destination ground station [4].

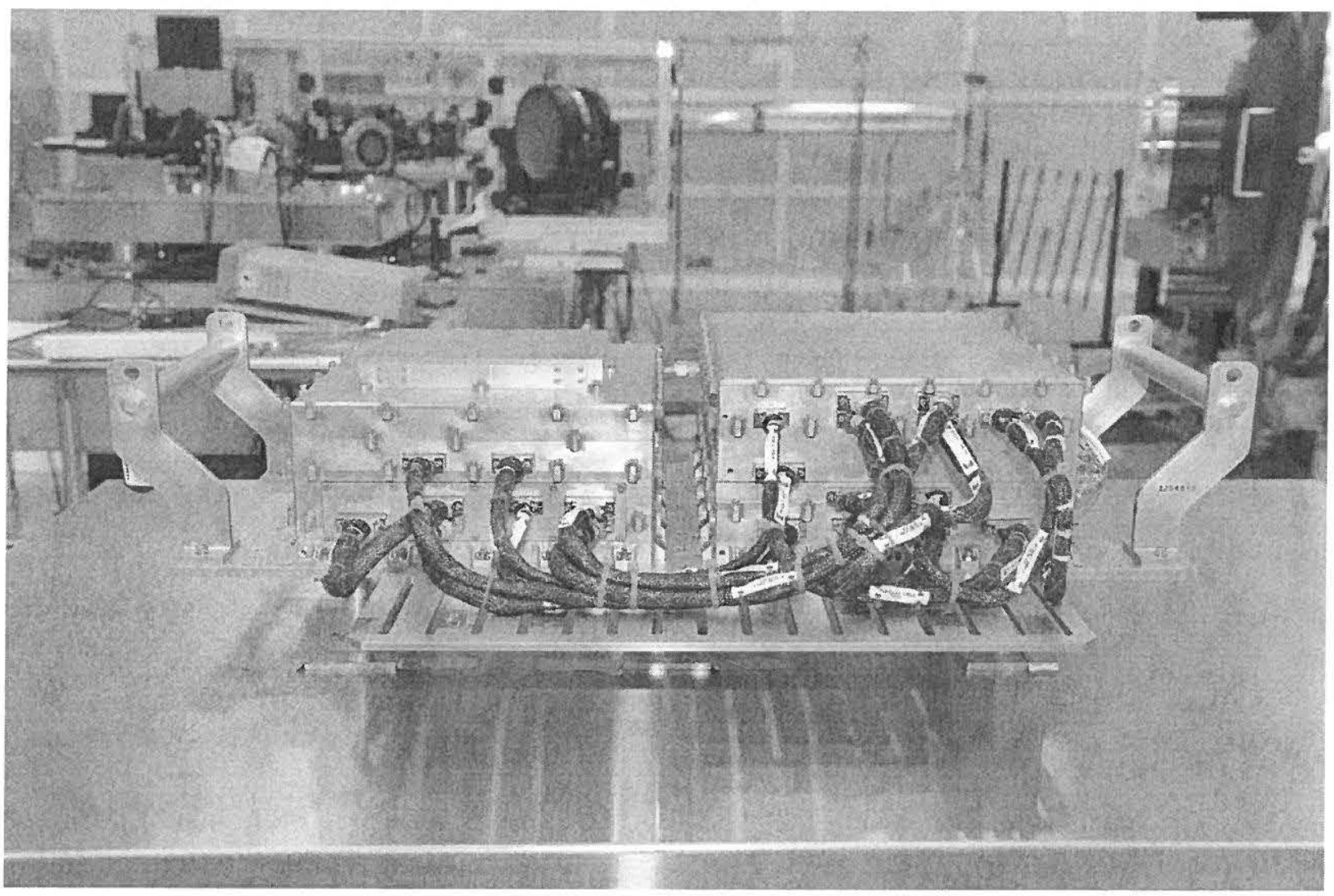

Fig. 3 Flight Modem 1

In addition to DPSK, each flight modem will also support pulse position modulation (PPM). The transmitter modulates the signal with a sequence of 16-ary PPM symbols (a signal pulse is placed in exactly one of each 16 temporal slots). The maximum PPM data rate is $311 \mathrm{Mbps}$. The transmitter utilizes the same $2.88 \mathrm{GHz}$ clock rate. When operating in PPM mode, the receive modem utilizes the same optical pre-amplification and optical filter as is used in DPSK. The optical signal is converted to an electrical signal by means of a photo-detector. The electrical signal in each slot is compared to a threshold (which can be varied to account for atmospheric turbulence) in a simple, yet sensitive PPM receiver implementation. This method leverages previous work performed by MIT Lincoln Laboratory [5].

\section{Space Switching Unit}

To be an optical relay demonstration, LCRD will create a relay connection between two optical ground stations or between a satellite in LEO and the ground. The Space Switching Unit interconnects the two flight optical terminals and the spacecraft's high rate RF system. In addition to real-time relay operations, the electronics will allow scenarios where one link uses DPSK signaling and the other PPM. A known challenge with optical communication through the atmosphere is the susceptibility to cloud cover. Thus the Space Switching Unit can use the high rate RF system in addition to an optical downlink when necessary. To support Delay Tolerant Networking (DTN) over the DPSK optical links, the Space Switching Unit will implement any required decoding and deinterleaving so the payload can process and route the data (at a rate less than the maximum DPSK throughput). The 
link operations will be configurable to allow support for a variety of scenarios.

\section{Secure Host Interface Module}

The secure host interface module (SHIM) isolates the LCRD flight payload, in an Information Assurance (IA) point of view, from the spacecraft. The addition of IA technology was a recent enhancement to LCRD and its purpose is to prevent unauthorized access, use, disclosure, disruption, modification, inspection, recording, or the destruction of information within the LCRD flight payload. IA gets the right information to the right people at the right time while protecting that information from eavesdropping or corruption. NASA's space communication links must be available when needed (e.g. be protected from a denial of service attack) and reliably transmit data (e.g. be protected from information manipulation). Some of NASA's communications, such as medical information and private astronaut communications, also need to be protected from eavesdropping. Thus IA is critical in preserving the integrity and confidentiality of future operational relay satellite systems; this is particularly needed for NASA's human exploration missions, such as the future Orion Crew Exploration Vehicle. With the SHIM, the LCRD flight payload could be hosted on an untrusted non-US government spacecraft and maintain complete information assurance.

\section{E. Payload Integration}

Integration and test of the LCRD flight payload has been occurring at NASA's Goddard Space Flight Center, and is near completion. Each optical terminal has been installed on the LCRD Support Assembly (LSA) provided by the spacecraft provider. Table 1 and Figure 4 identifies and shows the major components of the flight payload as installed on the LSA.

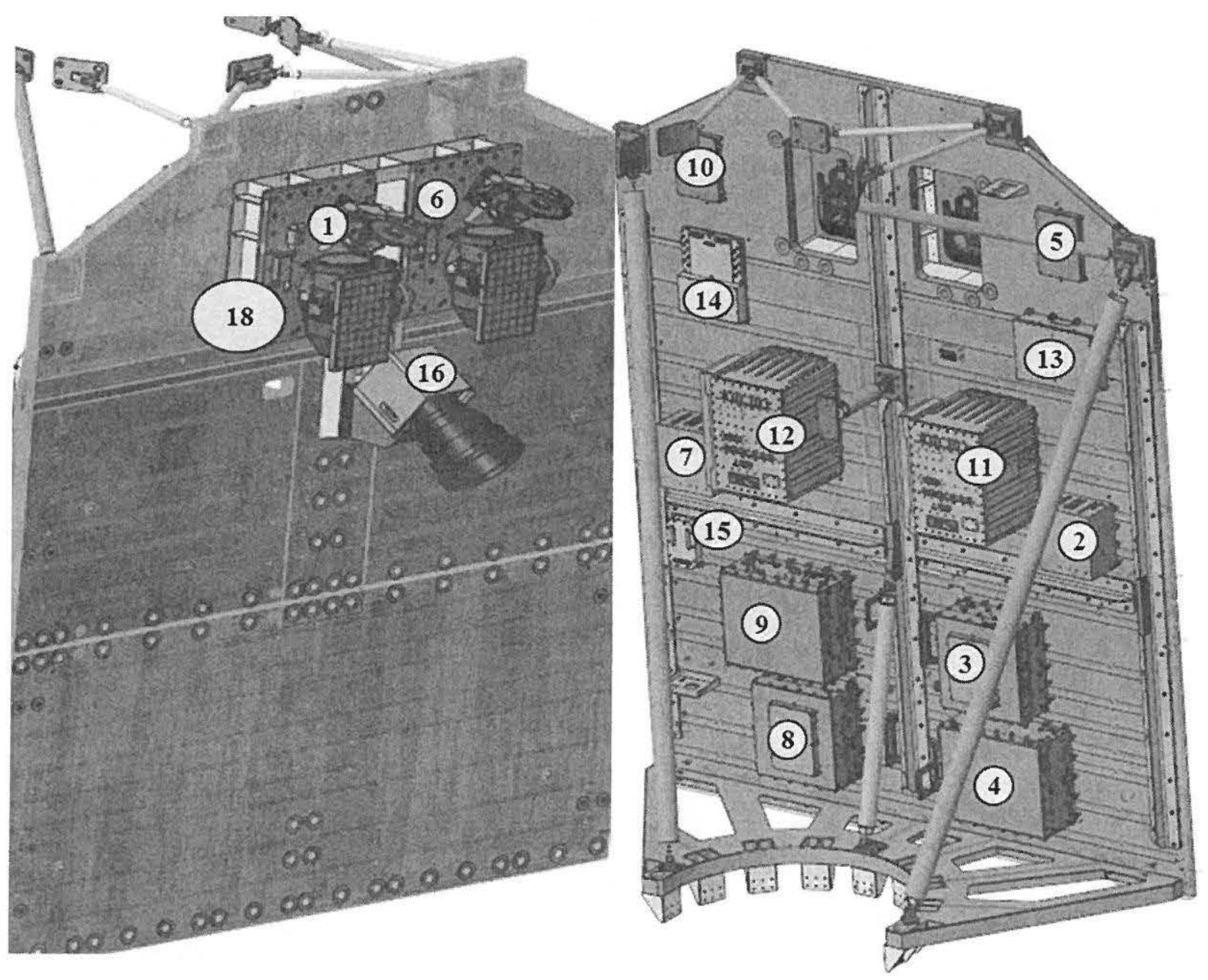

Fig. 4 LCRD Flight Payload Configuration (Outboard Side is on the Left) 


\begin{tabular}{|c|c|}
\hline ID \# & Payload Component \\
\hline 1 & Optical Module 1 \\
\hline 2 & Controller Electronics 1 \\
\hline 3 & Modem 1 Tower A \\
\hline 4 & Modem 1 Tower B \\
\hline 5 & Communications Splice Box 1 (CoSB 1) \\
\hline 6 & Optical Module 2 \\
\hline 7 & Controller Electronics 2 \\
\hline 8 & Modem 2 Tower A \\
\hline 9 & Modem 2 Tower B \\
\hline 10 & Communications Splice Box 2 (CoSB 2) \\
\hline 11 & Space Switching Unit A (Primary) \\
\hline 12 & Space Switching Unit B (Redundant) \\
\hline 13 & Secure Host Interface Module (SHIM) \\
\hline 14 & SV Protocol Coupler Router (PCR) \\
\hline 15 & SV Harness Interface Box \\
\hline 16 & SV Star Tracker \\
\hline 17 & LCRD Support Assembly (LSA) \\
\hline 18 & Isolation System \\
\hline
\end{tabular}

Table 1 LCRD Flight Payload Components

\section{Spacecraft}

The LCRD flight payload will fly on the Space Test Program Satellite 6 (STPSat-6). Space Test Program satellites are a series of spacecraft developed under a Department of Defense (DoD) program to field space capabilities quickly in response to emerging national needs. STPSat-6, which is managed by the Space Test Program office at Kirkland Air Force Base in Albuquerque, New Mexico, is scheduled to launch in summer 2019. The Space Test Program office is in the Advanced Systems and Development Directorate within the Space and Missile Systems Center of the U.S. Air Force. While the satellite is names STPSat-6, the entire mission is referred to as Space Test Program 3.

\section{A. Spacecraft}

The STPSat-6 spacecraft bus is being provided by Orbital ATK in Dulles, Virginia, and is based on a partially assembled satellite in storage; it was originally based on Orbital ATK's high-end modular A-500 bus. The satellite will be directly inserted into an orbit slightly above geostationary. STPSat-6 will carry several experiments into orbit in addition to LCRD. The primary payload is the Space and Atmospheric Burst Reporting System 3 from the National Nuclear Security Administration, which provides nuclear detonation detection and space environment data, and is designed to complement nuclear detonation detectors currently in orbit. The satellite will also host seven Department of Defense Space Experiments Review Board payloads from the Space Test Program office.

\section{B. High Data Rate RF}

When LCRD was originally conceived and approved, the project contained only two optical space terminals and two optical ground stations. This was considered to be the minimum configuration that would allow NASA to experiment and learn about a geosynchronous-Earth orbit (GEO)-based optical communications relay service. With two ground stations, NASA could simulate a handover from one ground station to another; in a different configuration, one ground station could act as the user and the other could act as the "receiving" ground station (i.e. as the optical trunkline from the relay satellite) [6].

This minimum configuration was never considered ideal, and it was recognized that a real operational demonstration required a user terminal in space communicating through LCRD to the ground. Furthermore, the minimum architecture would suffer from cloud coverage at either optical ground station. A benefit of an optical-tooptical relay is that it allows the trunkline to carry the same bandwidth as the link between the relay and the user. An actual optical relay would need to contend with inevitable cloud cover over one or both ground stations. The susceptibility to cloud cover, scintillation, scattering and other atmospheric effects are known challenges associated with optical communications. If a cloud were present, the optical trunkline could either wait for clouds to pass or 
switch to a different ground station that has a cloud-free line of sight; however, both of these options create a link outage.

A high data rate RF trunkline has been added to the LCRD architecture to provide more capability and alleviate concerns regarding lack of optical ground station availability due to cloud cover. NASA expects that future operational GEO relay satellites will have both optical and RF services. Optical communications would be used for extremely high data transfer where some latency might be acceptable; RF would provide high availability of services, but lower data transfer. For example, science instrument files, housekeeping files, software uploads and more can often be delayed as long as they are completely delivered. But most missions also have requirements for real-time or very low-latency delivery, such as commanding, telemetry, science alerts, voice, video, etc. [7]. In this way, the capabilities complement each other, enabling both high-volume data delivery and reliable coverage.

The STPSat-6 spacecraft is being augmented with a Ka-Band system that consists of two Software Defined Radios (SDRs). The system supports:

- Transmit up to $622 \mathrm{Mbps}$

- Receive up to $64 \mathrm{Mbps}$

- SpaceWire command, control, and telemetry to the spacecraft

- Two full duplex high speed Aurora interfaces (8B/10B at $3.125 \mathrm{Gbps})$

- Ability to be reconfigured on orbit

Each SDR on the spacecraft operate independently; one supports Right Hand Circular Polarization while the other supports Left Hand Circular Polarization. The system is equipped to receive and transmit in single or dual polarization modes at any time during the mission. The addition of high data rate RF makes the LCRD demonstration much more valuable to NASA in developing and experimenting with future concepts of operations.

\section{Ground Segment}

The LCRD Ground Segment is comprised of the LCRD Mission Operations Center (LMOC) and two optical ground stations. The LMOC will perform all scheduling, command, and control of the LCRD payload and the ground stations.

Each Earth ground station must provide three functions when communicating with one of the two optical communications terminals on the GEO spacecraft: receive the communications signal from the space terminal, transmit a signal to the space terminal, and transmit an uplink beacon beam so that the space terminal points to the correct location on the Earth. The receiver on Earth must provide a collector large enough to capture adequate power to support the data rate; couple this light onto low noise, efficient detectors while trying to minimize the coupled background light; and perform synchronization, demodulation, and decoding of the received waveform. The uplink beacon, transmitted from each Earth ground station, must provide a pointing reference to establish the GEO space terminal beam pointing direction. Turbulence effects dominate the laser power required for a ground-based beacon. Turbulence spreads the beam, reducing mean irradiance at the terminal in space, and causes fluctuations in the instantaneous received power.

\section{A. Spacecraft Operations at the White Sands Complex}

The STPSat- 6 spacecraft operations center and TT\&C RF ground station will be located at the White Sands Complex. The high data rate RF ground system will also be co-located there which will allow NASA to better gain insight into the operations of a spacecraft with an optical relay capability. LCRD payload operations in the LCRD mission operations center (LMOC), however, will be separated from spacecraft operations; this will facilitate the development of operations concepts that will not preclude future relay capabilities flying as hosted payloads.

LMOC will have access to real-time and predictive atmospheric data from both optical ground stations. When only one optical ground station is required for a particular communications pass, LMOC will be able to allocate a ground station based on predictive performance.

\section{B. Optical Ground Station 1}

NASA's Jet Propulsion Laboratory Optical Communications Telescope Laboratory (OCTL) will be used as LCRD Optical Ground Station 1. The OCTL is located on top of Table Mountain in the San Gabriel Mountains of southern California, and houses a 1-meter $\mathrm{f} / 75.8$ coudé focus telescope (shown in Figure 5). The large aperture readily supports the high-data-rate differential phase shift keying (DPSK) and pulse position modulation (PPM) downlinks from the LCRD space terminal with adequate link margin. Required to operate $24 / 7$, in the presence of winds and as close as 5 
degrees solar angles, the OCTL telescope will be enclosed in a temperature-controlled dome with a transparent window to allow laser beam and radar transmission [8].

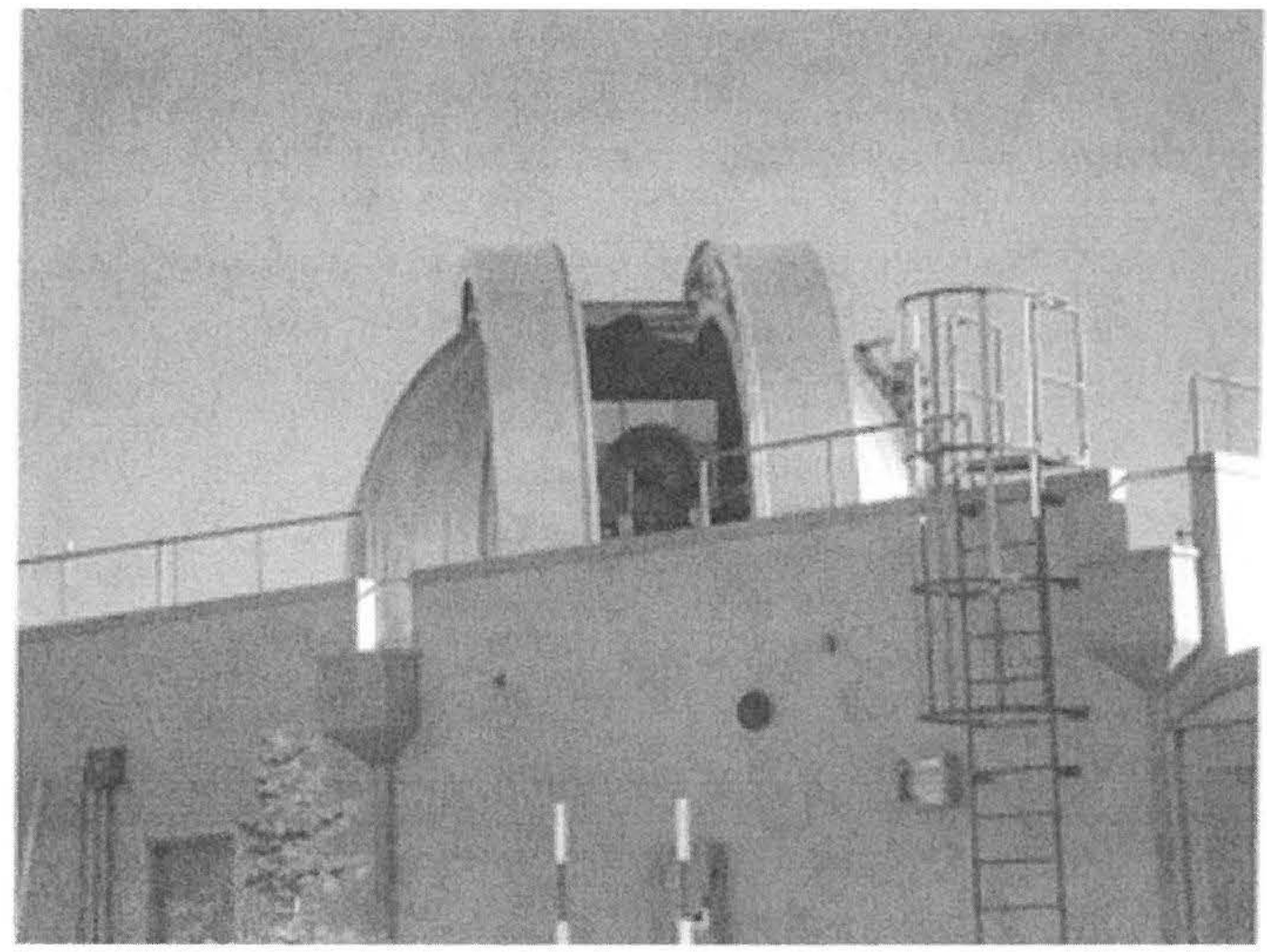

Fig. 5 Optical Ground Station 1

\section{Optical Ground Station 2}

Optical Ground Station 2 is located at the Air Force's Maui Optical and Supercomputing Space Surveillance Complex on top of Haleakala in Maui, Hawaii (Figure 6). It has a 60-centimeter receive aperture and a 15-centimeter transmit aperture; both are located within an approximately 5.5-meter diameter dome on the roof (Figure 7). Elevation over azimuth gimbals support the two telescopes. The dome provides an enclosed temperature-stabilized environment with a steerable window for day and night operations. 


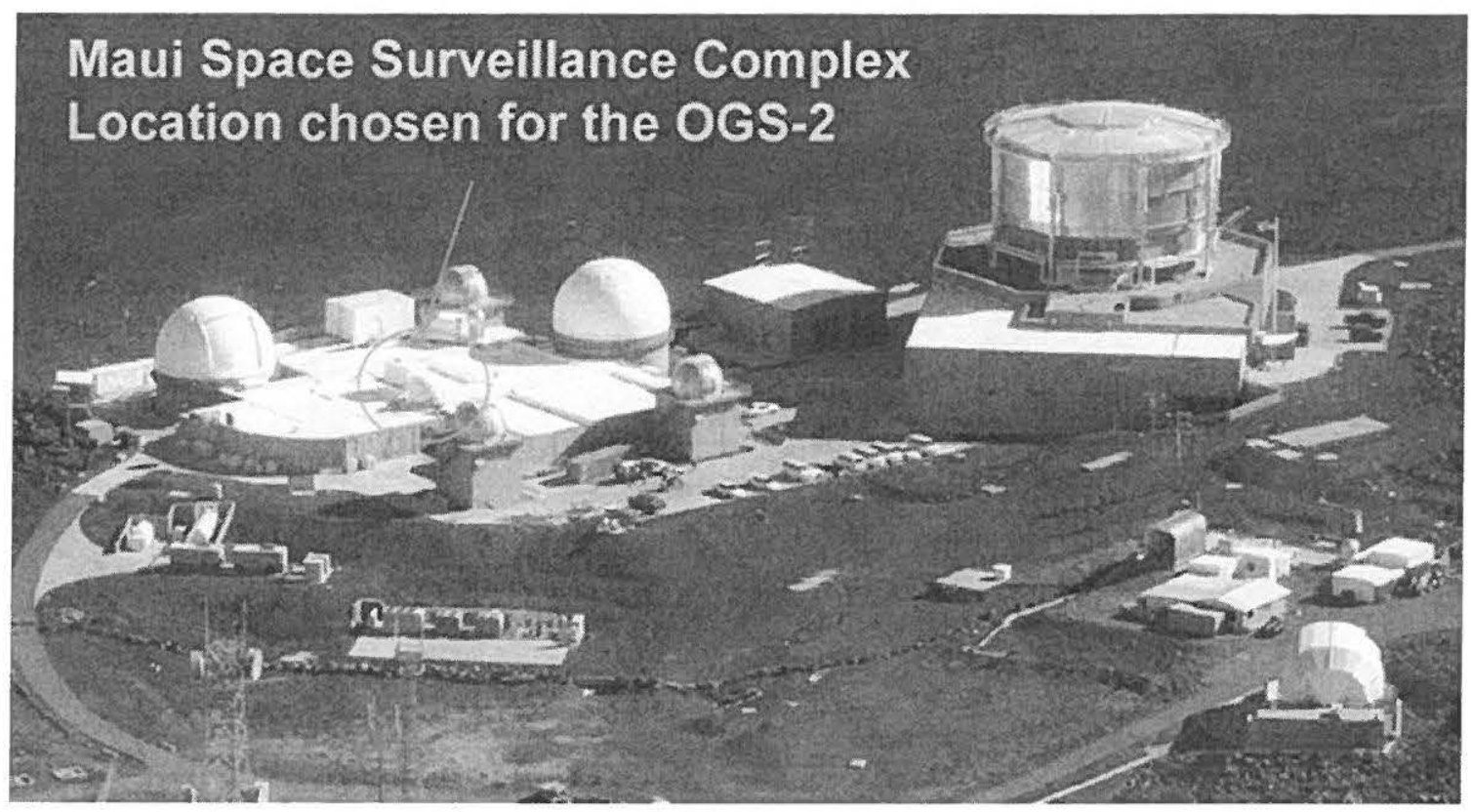

Fig. 6 Optical Ground Station 2 Location

The laser subsystem consists of a custom photonics assembly that produces a low-power $(<10$ milliwatt), fibercoupled optical signal, followed by a commercially obtained high-power optical amplifier. The fiber amplifier can produce up to 10 watts of optical power, but will be limited by software to a maximum power of 7.3 watts during operation. After accounting for transmission loss of the transmit telescope and the window in the dome, the maximum power emitted into free space outside the dome is 5.4 watts. Figure 8 shows the rack room where most of the electronics are housed in the ground station.

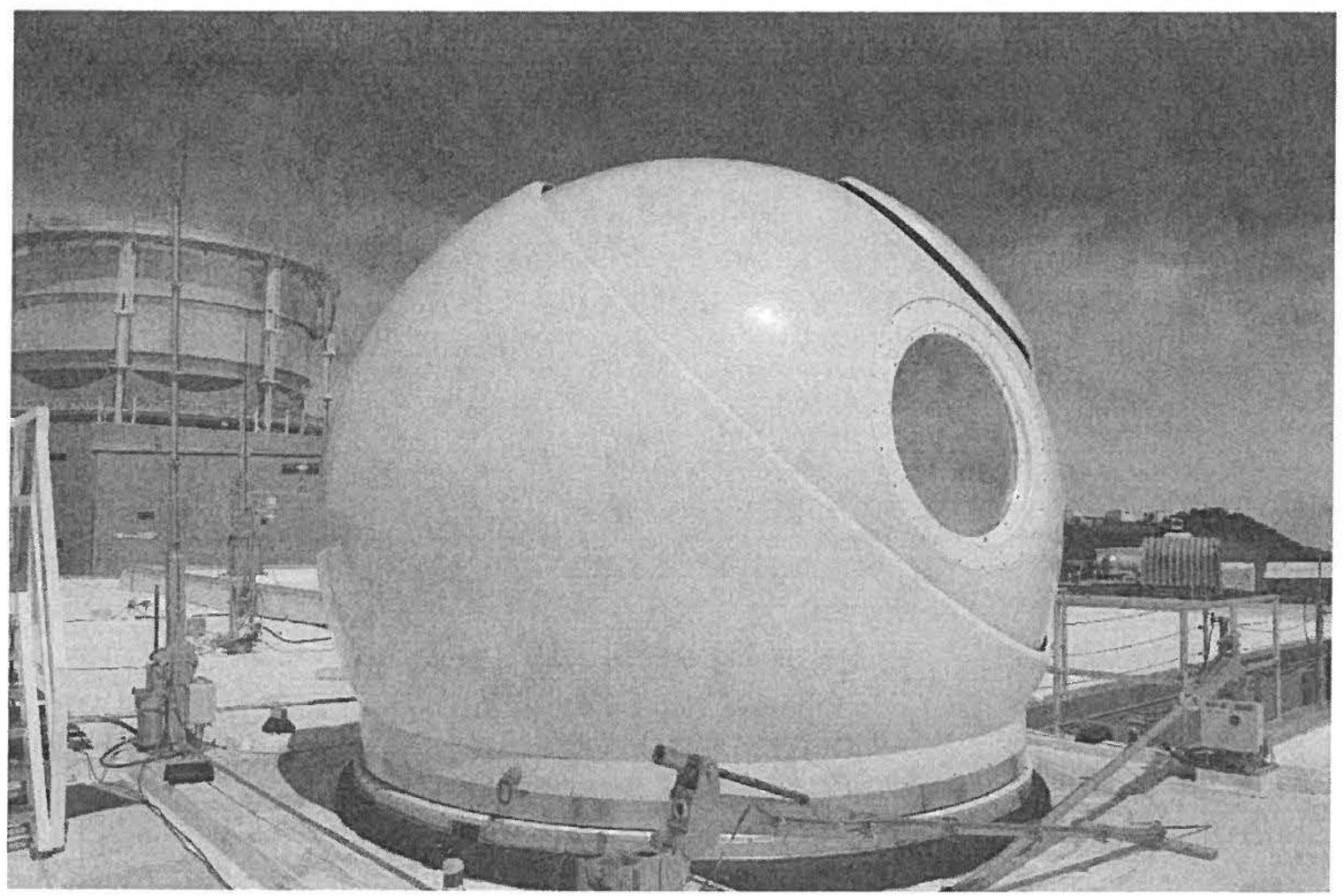


Fig. 7 Telescope Dome at Optical Ground Station 2

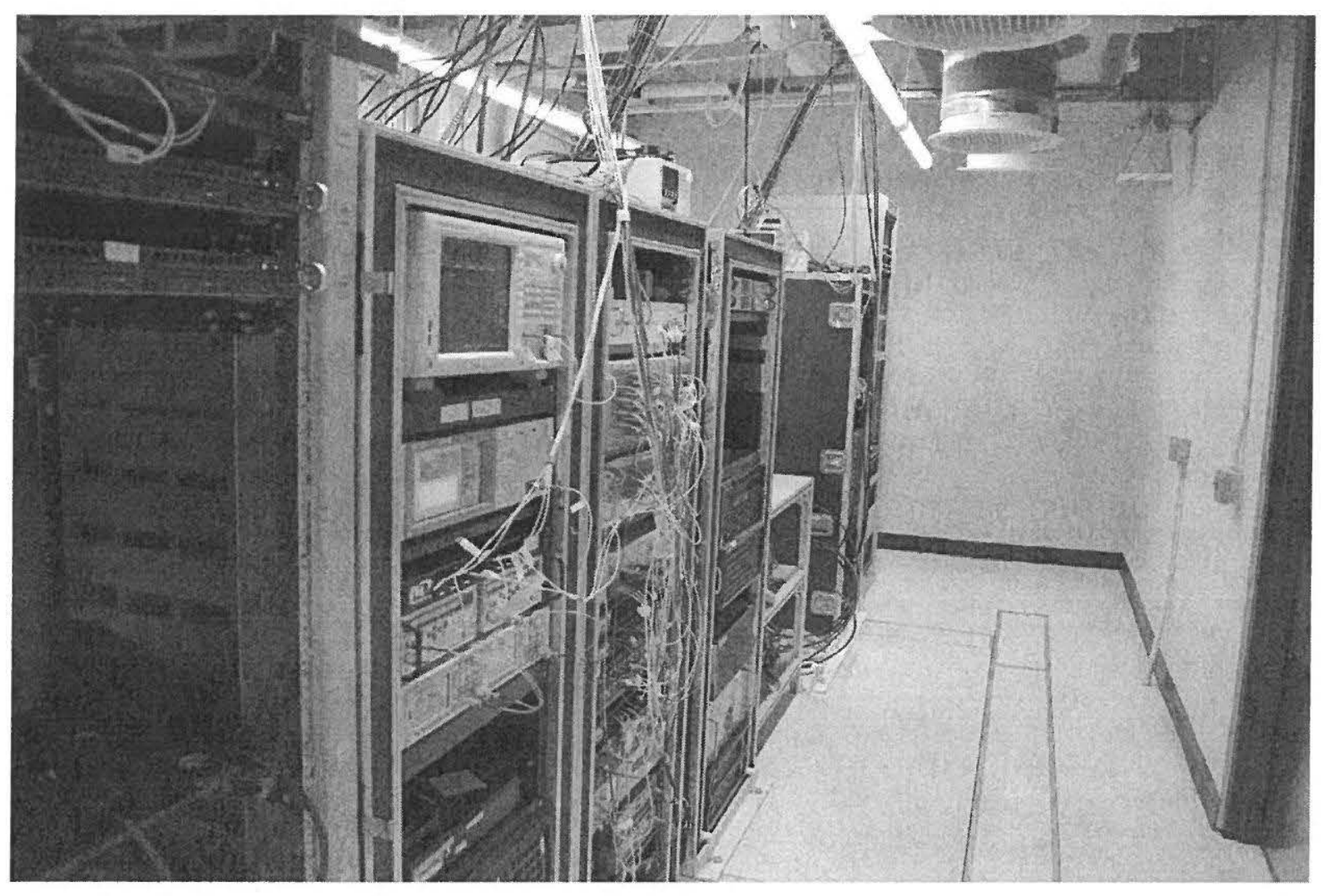

Fig. 8 Rack Room at Optical Ground Station 2

\section{Conclusion}

LCRD has evolved since it was originally proposed. The current architecture more closely resembles what is expected in a future operational relay satellite. The integration and testing of the LCRD flight payload at NASA's Goddard Space Flight Center is going well, and NASA is looking forward to a summer 2019 launch. Both Optical Ground Stations and the LCRD Mission Operations Center are on schedule. As a hosted payload on the STPSat-6 spacecraft, LCRD has already provided NASA with knowledge and experience about the advantages of disadvantages of this approach. The value of LCRD will be further enhanced once an LCRD-compatible user terminal is installed on the International Space Station in 2021.

\section{Acknowledgments}

The work described in this paper was carried out at NASA's Goddard Space Flight Center; at Lincoln Laboratory*, Massachusetts Institute of Technology; and at the Jet Propulsion Laboratory, California Institute of Technology, under contract with NASA. It is funded by NASA's Space Technology Mission Directorate's Technology Demonstration Missions (TDM) program and NASA's Human Exploration and Operations (HEO) Mission Directorate's Space Communications and Navigation ( $\mathrm{SCaN}$ ) program.

\section{References}

\footnotetext{
* This work was sponsored by NASA Goddard Space Flight Center under Air Force Contract FA8721-05-C-0002. The opinions, interpretations, conclusions, and recommendations are those of the author and not necessarily endorsed by the United States Government.
} 
1. Edwards, B.L.; Israel, D.J.; Vithlani, S.K., "Changes to NASA's Laser Communications Relay Demonstration Project", SPIE Photonics West Conference, 2018

2. Wang, J.P.; Magliocco, R.J.; Spellmeyer, N.W.; Rao, H.; Kochhar, R.; Caplan, D.O.; Hamilton, S.A.; , "A consolidated multi-rate burst-mode DPSK transmitter using a single Mach-Zehnder modulator," Optical Fiber Communication Conference and Exposition (OFC/NFOEC), 2011 and the National Fiber Optic Engineers Conference, vol., no., pp.1-3, 6-10 March 2011

3. Caplan, D.O.; Rao, H.; Wang, J.P.; Boroson, D.M.; Carney, J. J.; Fletcher, A.S.; Hamilton, S.A.; Kochhar, R.; Magliocco, R.J.; Murphy, R.; Norvig, M.; Robinson, B.S.; Schulein, R.T.; Spellmeyer, N.W.; , "Ultra-wide-range multi-rate DPSK laser communications," Lasers and Electro-Optics (CLEO) and Quantum Electronics and Laser Science Conference (QELS), 2010 Conference on, vol., no., pp.1-2, 16-21 May 2010

4. Shoup, R.; List, N.; Fletcher, A.; Royster, T.; , "Using DVB-S2 over asymmetric heterogeneous optical to radio frequency satellite links," Military Communications Conference, 2010 - MILCOM 2010 , vol., no., pp.785-790, Oct. 31 2010-Nov. 32010

5. N. Spellmeyer, S. Bernstein, D. Boroson, D. Caplan, A. Fletcher, S. Hamilton, R. Murphy, M. Norvig, H. Rao, B. Robinson, S. Savage, R. Schulein, M. Stevens, and J. Wang, "Demonstration of Multi-Rate Thresholded Preamplified 16-ary Pulse-Position-Modulation," in Optical Fiber Communication Conference, OSA Technical Digest (CD) (Optical Society of America, 2010), paper OThT5.

6. Edwards, Bernard, David Israel, Armen Caroglanian, James Spero, Tom Roberts, and John Moores. "A Day in the Life of the Laser Communications Relay Demonstration Project." in 14th International Conference on Space Operations, p. 2590. 2016.

7. Interagency Operations Advisory Group, "Optical Link Study Group Final Report,"IOAG.T.OLSG.2012. 5 June 2012

8. K. E. Wilson, J. Wu, N. Page, M. Srinivasan, "The JPL Optical Communications Telescope Laboratory (OCTL), Test Bed For The Future Optical Deep Space Network" JPL, Telecommunications and Data Acquisition Progress Report 142 -153 February 2003. 